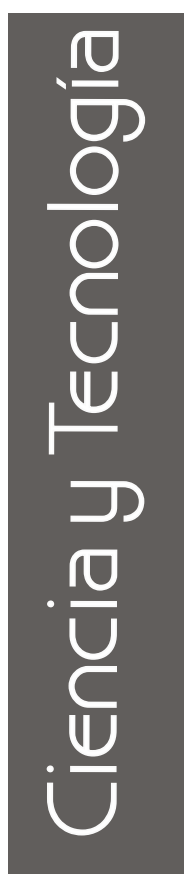

\title{
Levantamiento y Actualización del Modelo de una Red Primaria de Distribución
}

\author{
Gladys Caicedo* \\ Carlos A. Lozano** \\ Jhon Edwin Candelo*** \\ Diego F. Figueroa***
}

* M.sc. Profesora Titular de la Escuela de Ingeniería Eléctrica y Electrónica - Facultad de Ingeniería - Universidad del Valle Santiago de Cali - Colombia.

e-mail:glacadel@eiee.univalle.edu.co

** Ph.D. Profesor Asociado Escuela de Ingeniería Eléctrica y Electrónica - Universidad del Valle - Santiago de Cali Colombia. e-mail:clozano@eiee.univalle.edu.co

*** Ingeniero Electricista - Empresa de Energía del Pacífico EPSA - Santiago de Cali - Colombia. E-mail:jecandelo@epsa.com.co

*** Ingeniero Electricista - Empresa de Energía del Pacífico EPSA - Santiago de Cali - Colombia.

E-mail:dfigueroa@epsa.com.co

Fecha de recepción: Moviembre 8 de 2002 Fecha de aprobación: Marzo 26 de 2003

\section{RESUMEN}

En este artículo se presenta la metodología que permite obtener el modelo actualizado de una red primaria de distribución. En la primera parte se describe el procedimiento realizado para el cálculo de la resistencia y la reactancia en cada rama de la red a partir de los datos del sistema de información geográfico ( $S I G$ ) y las aproximaciones realizadas en el cálculo de cada parámetro. Además se describe la metodología del levantamiento de la información del SIG para obtener la configuración de la red. Finalmente se muestra la estructura del programa de computador desarrollado para el cálculo automático tanto de los parámetros como de la configuración. 
La herramienta computacional se probó para el sistema de distribución de EPSA en el municipio de Buenaventura, el cual es un sistema que tiene aproximadamente 6000 nodos y 1300 usuarios. Los resultados obtenidos en precisión y rapidez de respuesta son buenos y han sido utilizados en estudios de pérdidas.

Palabras Clave: Sistema de distribución, Modelo red primaria, Sistema de información geográfico.

\section{ABSTRACT}

This paper shows a methodology that allows to obtain an up to date model of a primary distribution network. In the first part, the procedure used for the resistance and reactance calculation of each branch from the Geographic Information System data is described. Also, the approximations used in calculating each parameter are mentioned. Then, the methodology for obtaining the information from the GIS to obtain the network configuration is described and finally, the software structure developed for the automatic calculations of the parameters and the configuration is shown. The computational tool was tested in a real distribution system, in a medium size town (Buenaventura) that has about 6000 buses and 1300 customers, which belongs to a regional utility (EPSA:Empresa de Energía del Pacifico ESP).

Key Words: Distribution system, primary distribution network model, geographic information system (GIS).

\section{INTRODUCCIÓN}

El actual esquema competitivo en el sector eléctrico, exige de las empresas la implementación de programas para mejorar su desempeño en todas las áreas. En lo que se refiere a los sistemas de distribución se requiere mejorar aspectos como: restauración del servicio, balance de cargas y reducción de pérdidas.
En el análisis de sistemas de distribución se requiere el desarrollo de herramientas computacionales, tales como flujos de carga, reconfiguración de redes, restauración de alimentadores, los cuales requieren modelos precisos en los diferentes elementos del sistema (red primaria, transformadores de distribución, red secundaria y la demanda).

El modelo de una red primaria se obtiene al calcular los valores de resistencia y reactancia por cada rama y al determinar la configuración de la red.

Aunque desde el punto de vista matemático el modelo es sencillo, desde el punto de vista práctico obtenerlo y actualizarlo es complejo por los siguientes aspectos:

- Los parámetros de los modelos (resistencia y reactancia) de la red cambian continuamente, debido a que la red es dinámica.

- La red de distribución primaria es demasiado grande.

Por esto, aunque existe un procedimiento estandar para el levantamiento de los datos de campo de los elementos de la red, tales como: calibre de conductor, tipo de conjunto, coordenadas de los apoyos, etc., se requiere adicionalmente realizar cálculos para obtener los valores de los parámetros en una red de distribución real. Algunas herramientas computacionales existentes como las utilizadas en flujos de carga de la EPRI y en MEPLAM, permiten el cálculo de los parámetros $R$ y $X$ por ramal, sin utilizar toda la información requerida, permitiendo un cálculo aproximado.

En este artículo se presenta una metodología y la estructura de una herramienta computacional para obtener el modelo preciso y actualizado de la red primaria, a partir de los datos levantados de la red de distribución, realizado con el sistema de información geográfica (SIG) [1]. 


\section{CÁLCULO DEL MODELO}

El modelo de una red primaria de distribución consta de dos partes: una la obtención de los parámetros de resistencia y reactancia en cada una de las ramas y la otra es la configuración de toda la red. A continuación se describe el procedimiento para obtener ambas partes.

\subsection{Cálculo de los parámetros}

El modelo matemático de cualquier rama en una red de distribución es:

$$
R+J X L
$$

Esto significa que el modelo queda definido, cuando los valores de R y XL sean cuantificados.

\subsubsection{Datos requeridos para el cálculo de la resistencia}

La resistencia es un parámetro inherente al conductor y es este elemento el que produce las pérdidas de energía activa en la red primaria. El cálculo de la resistencia se realiza mediante la expresión [3] :

$$
R=R C A^{*} L
$$

Donde:

RCA: Es la resistencia de corriente alterna que depende de la temperatura y del material del conductor.

\section{L: $\quad$ Es la longitud de la rama en [km]}

En la Tabla 1 se ilustran los datos de los valores de $R C A$ entregados por el fabricante.

Este valor debe corregirse a la temperatura de trabajo requerida, mediante la siguiente expresión:

$$
R C A\left(t_{2}\right)=\frac{T+t_{2}}{T+t_{1}} * R C A\left(t_{1}\right)
$$

Donde:

t1: Temperatura a la cual el fabricante calcula la resistencia del conductor.

t2: Temperatura a la cual se desea calcular una nueva resistencia para el conductor.

$R C A(t 1)$ : Resistencia a la temperatura dada por el fabricante.

$R C A(t 2)$ : Resistencia calculada a una nueva temperatura.

$T$ : temperatura de cocido del material.

\begin{tabular}{|c|c|c|c|c|c|}
\hline \multirow{2}{*}{ Calibre } & \multicolumn{3}{|c|}{$R C A / K m$} & $R C D / K m$ & $D s$ \\
\cline { 2 - 5 } & $25^{\circ} \mathrm{C}$ & $50^{\circ} \mathrm{C}$ & $75^{\circ} \mathrm{C}$ & $20^{\circ} \mathrm{C}$ & {$[\mathrm{m}]$} \\
\hline 6 & 2,14 & 2,44 & 2,68 & 2,113 & 0,0024 \\
\hline 4 & 1,35 & 1,56 & 1,71 & 1,327 & 0,0026 \\
\hline 2 & 0,85 & 1,01 & 1,10 & 0,834 & 0,0025 \\
\hline $1 / 0$ & 0,53 & 0,65 & 0,71 & 0,524 & 0,0027 \\
\hline $2 / 0$ & 0,42 & 0,53 & 0,58 & 0,416 & 0,0031 \\
\hline $3 / 0$ & 0,33 & 0,42 & 0,47 & 0,330 & 0,0036 \\
\hline $4 / 0$ & 0,27 & 0,35 & 0,38 & 0,261 & 0,0049 \\
\hline 336 & 0,17 & 0,18 & 0,20 & 0,168 & 0,0148 \\
\hline
\end{tabular}

Tabla 1. Valores RCA entregados por el fabricante

\subsubsection{Longitud de la rama o distancias entre apoyos}

El valor de la longitud entre apoyos se obtiene de la información de las coordenadas en tres dimensiones levantadas por el SIG para cada uno de los apoyos entre ramas, con estos datos se realiza la diferencia entre las coordenadas físicas. En la Figura 1 se ilustran las mediciones realizadas por el SIG para un par de apoyos.

Mediante la ecuación (4) se calcula la longitud entre apoyos, teniendo en cuenta todas las coordenadas. 
Leeq $=$ Le2-Le1

Distancia equivalente al este entre los apoyos.

Lneq $=\operatorname{Ln} 2-\operatorname{Ln} 1$

Distancia equivalente al norte entre los apoyos.

Lheq $=$ Lh2-Lh1

Distancia equivalente al nivel entre los apoyos.

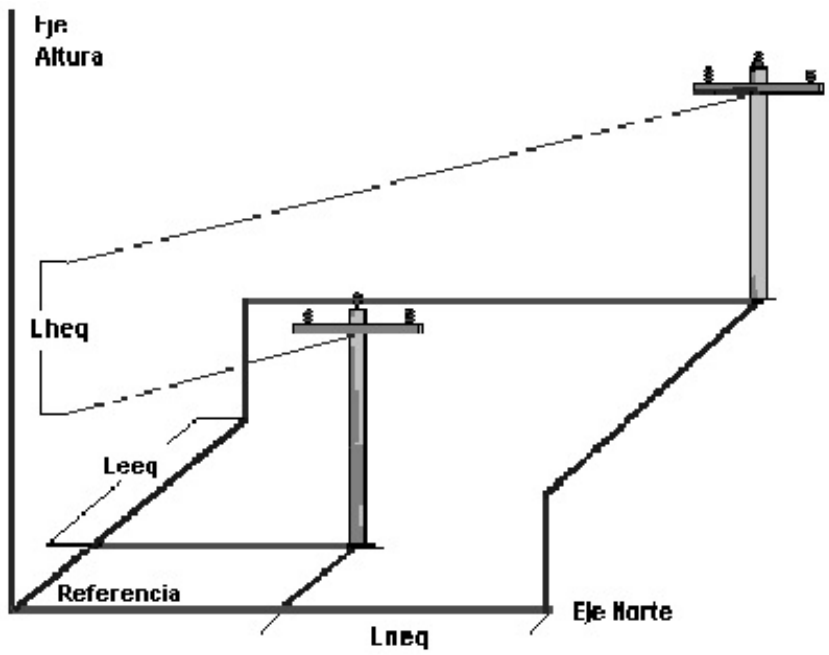

Figura 1. Cálculo de la longitud entre apoyos

\subsection{Datos requeridos para el cálculo de la reactancia.}

La reactancia inductiva se origina del fenómeno electromagnético del funcionamiento de la línea y este elemento es el que produce las pérdidas de energía reactiva en la red primaria. La reactancia se calcula mediante la expresión [3]:

$$
X_{\mathrm{L}}=4 \operatorname{TTf} * 10^{-7} \ln \frac{\mathrm{D}_{\mathrm{eq}}}{\mathrm{DS}} * \mathrm{~L}
$$

Donde:

Ds : Diámetro medio geométrico del conductor.

Este valor es entregado por el fabricante,

Tabla1.

F: Frecuencia

L: $\quad$ Longitud entre apoyos del ramal.

Deq:Espaciamiento equivalente entre los Conductores.
El valor de Deq se calcula mediante la ecuación (6), [3].

$$
D_{\text {eq }}=\sqrt[3]{D_{12} D_{23} D_{31}}
$$

Donde:

D12 Distancia entre fase 1 y 2

D23 Distancia entre fase 2 y 3

D31 Distancia entre fase 3 y 1

En la Tabla 2 se ilustra el valor Deq para algunos conjuntos de la red de distribución de Buenaventura.

\begin{tabular}{|l|c|c|c|c|c|}
\hline Calibre & $\begin{array}{c}\text { Mo. De } \\
\text { Fases }\end{array}$ & $\begin{array}{l}\text { D12 } \\
{[\mathrm{m}]}\end{array}$ & $\begin{array}{l}\text { D23 } \\
{[\mathrm{m}]}\end{array}$ & $\begin{array}{c}\text { D13 } \\
{[\mathrm{m}]}\end{array}$ & $\begin{array}{c}\text { Dequi } \\
{[\mathrm{m}]}\end{array}$ \\
\hline B151A & 3 & 1,50 & 0,70 & 2,20 & 1,3219 \\
\hline B152A & 3 & 1,50 & 0,70 & 2,20 & 1,3219 \\
\hline B152BA & 3 & 1,50 & 0,70 & 2,20 & 1,3219 \\
\hline B156A & 3 & 1,50 & 0,70 & 2,20 & 1,3219 \\
\hline
\end{tabular}

Tabla 2. Espaciamientos de conductores según el conjunto

\subsection{Aproximación en el Cálculo de los Parámetros}

En el programa desarrollado se realizaron las siguientes aproximaciones:

1. Mo se calculó la resistencia y la reactancia para las siguientes uniones de la red:

- Cruces aéreos.

- Transformadores y fusibles.

-Fusibles y líneas.

-Monopolares y transformadores subterráneos

Esto debido a que la longitud es muy corta, $30 \mathrm{~cm}$ en comparación con la distancia de 30 m que existen entre apoyos.

2. Cuando el espaciamiento del conjunto de origen es diferente al conjunto de llegada. Para solucionar dicho inconveniente se optó por fraccionar el ramal en dos partes, una mitad se calcula con los espaciamientos del primer 
conjunto y la otra mitad con los espaciamientos del segundo. En la Figura 2 se ilustran ambos espaciamientos.

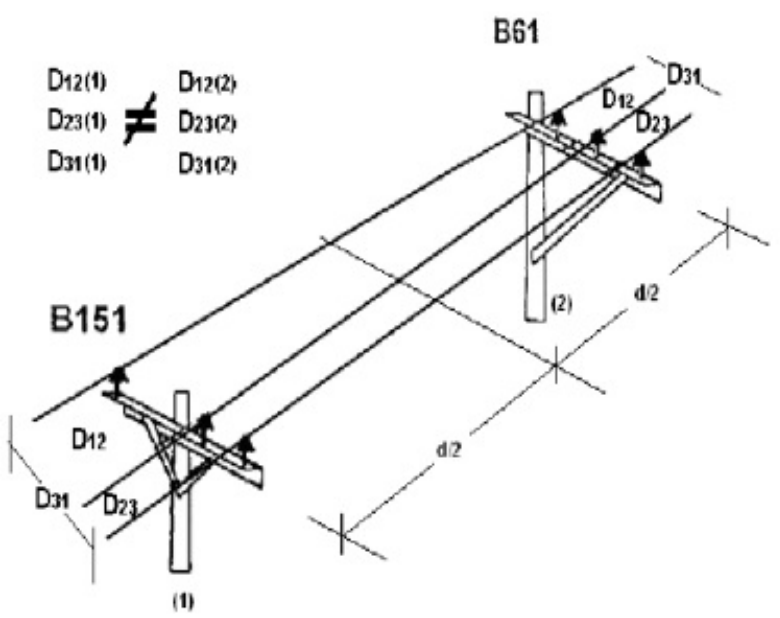

Figura 2. Diferencias de distancia entre los conductores en los conjuntos.

3. Cuando hay un apoyo y un cruce aéreo, se asume que el cruce aéreo tiene el mismo espaciamiento que el conjunto de origen.

El valor de la longitud $L$ para el cálculo de la reactancia, es el mismo que se efectuó para el cálculo de la resistencia.

\section{LEVANTAMIENTO DE LA TOPOLOGÍA DE LARED}

La información de topología de la red del sistema de distribución de Epsa, es actualizada mediante un procedimiento estandar, realizado por SIG [2]. Esta información es levantada de acuerdo al siguiente procedimiento:

- Elementos: Se debe tener disponible planos, formatos de nodos, tablas de circuitos, normas de diseño y GPS's .

- Identificación de los planos: Se debe observar en los planos las características del terreno, la red vial, la infraestructura física y sitios claves para el recorrido. Se deben localizar la subestación, recorrido principal y sitios de bifurcación.

- Recorrido del circuito: Partiendo de la subestación se debe identificar el nombre y código del circuito, definir los nodos eléctricos, los puntos de apertura y cierre, localización de seccionadores y puntos de frontera con otros circuitos. Debe procurarse seguir el circuito desde la subestación hasta el final y no al contrario debido al seguimiento del faseado.

- Orden en la toma de datos: Para que el levantamiento de los datos sea de mayor facilidad para la transcripción en computador, se debe llevar un orden específico en las tablas.

- Coincidencia de datos: Las tablas del levantamiento de datos de los circuitos deben coincidir en los números de los apoyos, sus niveles y las fases tomadas en cada tramo. En caso de error se debe verificar cuidadosamente en el sitio donde se tomaron los datos.

La recolección de los datos se realiza en tres tablas: Tabla de nodos eléctricos, conjuntos y dispositivos, tabla de conductores primarios y tabla de nodos físicos.

\subsection{Tabla de nodos eléctricos, conjuntos y dispositivos eléctricos}

Los nodos eléctricos son los puntos de contacto de los diferentes tramos del conductor. Los conjuntos se describen mediante códigos, de acuerdo a la norma de EPSA, que indican los elementos utilizados en la red. En la Tabla 3 se ilustran los datos de los puntos eléctricos y sus dispositivos.

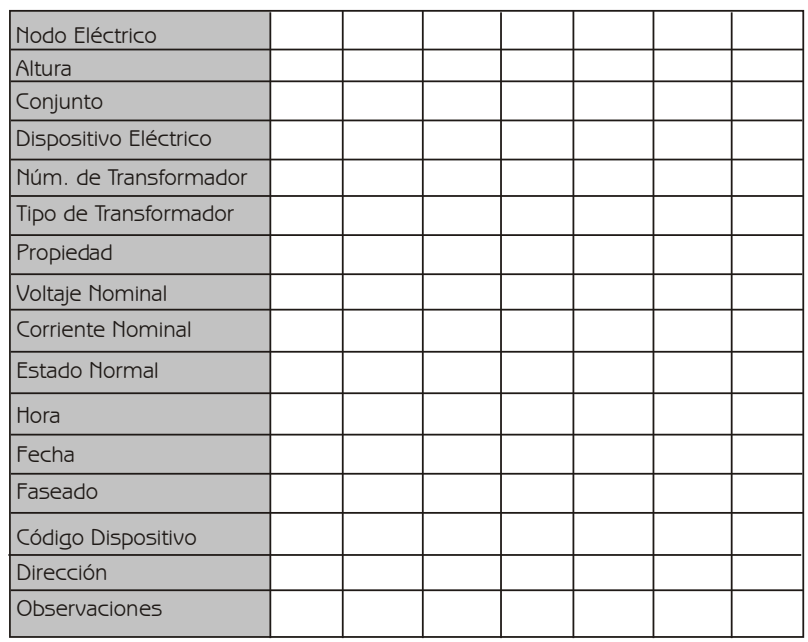

Tabla 3. Modos eléctricos y conjuntos 


\subsection{Tabla de conductores primarios}

En esta tabla se especifica el tipo de red (Aérea o Subterránea), el calibre y material de los conductores primarios y el faseado. En la Tabla 4 se ilustran los datos a recopilar.

\begin{tabular}{|c|c|c|c|c|}
\hline $\begin{array}{c}\text { Nodo } \\
\text { Eléctrico } \\
\text { Actual }\end{array}$ & $\begin{array}{c}\text { Modo } \\
\text { Eléctrico } \\
\text { Anterior }\end{array}$ & $\begin{array}{c}\text { Tipo } \\
\text { A: Áéreo }\end{array}$ Subterrán. & $\begin{array}{c}\text { Código } \\
\text { Primario }\end{array}$ & Fases \\
\hline & & & & \\
\hline & & & & \\
\hline & & & & \\
\hline
\end{tabular}

Tabla 4. Conductores Primarios

De esta tabla se obtiene la configuración de la red por circuito.

\subsection{Tabla de nodos físicos}

Se describen las características de los nodos físicos, coordenadas tomadas por el GPS y descripción de elementos técnicos. En la Tabla 5 se muestran algunos de estos datos.

\begin{tabular}{|l|l|l|l|l|l|}
\hline MSM & & & & & \\
\hline MORTE & & & & & \\
\hline OESTE & & & & & \\
\hline ID_LAMP & & & & & \\
\hline TL & & & & & \\
\hline TV & & & & & \\
\hline PARARRAYOS & & & & & \\
\hline HILO GUARDA & & & & & \\
\hline RETEMIDA & & & & & \\
\hline ID_MODO FISICO & & & & & \\
\hline MUMICIPIO & & & & & \\
\hline SECTOR & & & & & \\
\hline CÓDIGO SUB. & & & & & \\
\hline
\end{tabular}

Tabla 5. Modos Físicos

\subsection{Ejemplo: División de ramales.}

La Figura 3 muestra una división del ramal principal. Para hacerse el levantamiento de datos se debe seguir la ruta indicada, en la Tabla 6 se muestra su levantamiento.

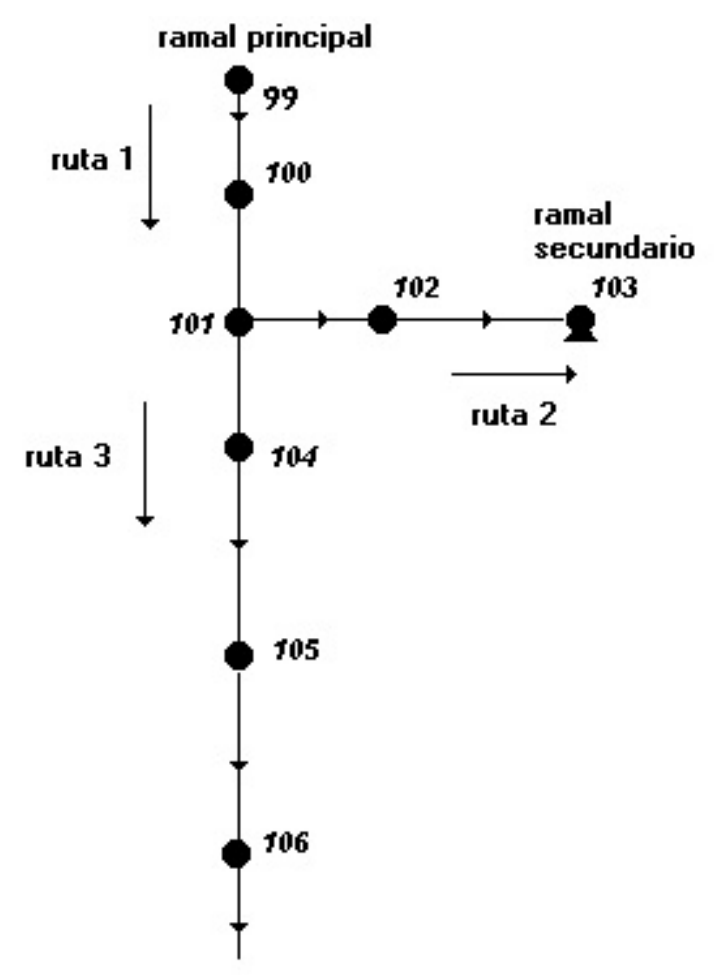

Figura 3. División de Ramal

\begin{tabular}{|l|c|c|c|}
\hline $\begin{array}{c}\text { Modo } \\
\text { Eléctrico } \\
\text { Actual }\end{array}$ & $\begin{array}{c}\text { Modéctrico } \\
\text { Anterior }\end{array}$ & $\begin{array}{c}\text { Tipo } \\
\text { A: Aéreo } \\
\text { S: Sbuterr. }\end{array}$ & Fases \\
\hline $100-1$ & $99-1$ & A & RST \\
\hline $101-1$ & $100-1$ & A & RST \\
\hline $102-1$ & $101-1$ & A & RST \\
\hline $103-1$ & $102-1$ & A & RST \\
\hline $104-1$ & $103-1$ & A & RST \\
\hline $105-1$ & $104-1$ & A & RST \\
\hline $106-1$ & $105-1$ & A & RST \\
\hline
\end{tabular}

Tabla 6. Levantamiento de Datos de un Ramal

Se toma el ramal principal en la parte superior (apoyos 99, 100 y 101). En este caso el ramal secundario corresponde al ramal más corto, y se escoge el ramal secundario como segundo camino (apoyos 102 y 103). Cuando se ha 
terminado el levantamiento en este ramal secundario se continúa con el ramal principal inferior (apoyos 104, 105 y 106).

\section{DESCRIPCIÓM DEL PROGRAMA PARA CALCULAR LOS PARÁMETROS}

En la Figura 4 se ilustra el diagrama ambiente en que fue desarrollada la herramienta computacional.

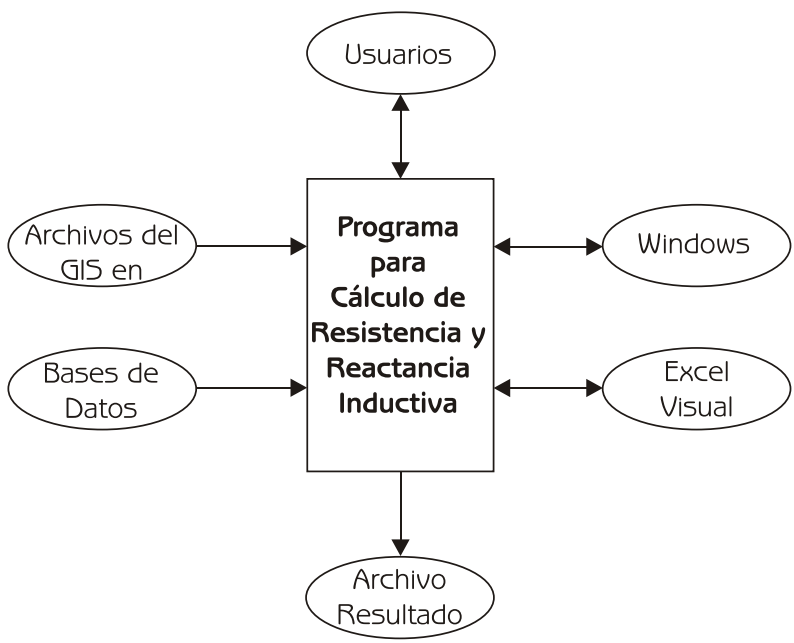

Figura 4. Diagrama Ambiente del Programa

En la Figura 5 se ilustran los datos entrada y los resultados obtenidos del programa desarrollado.

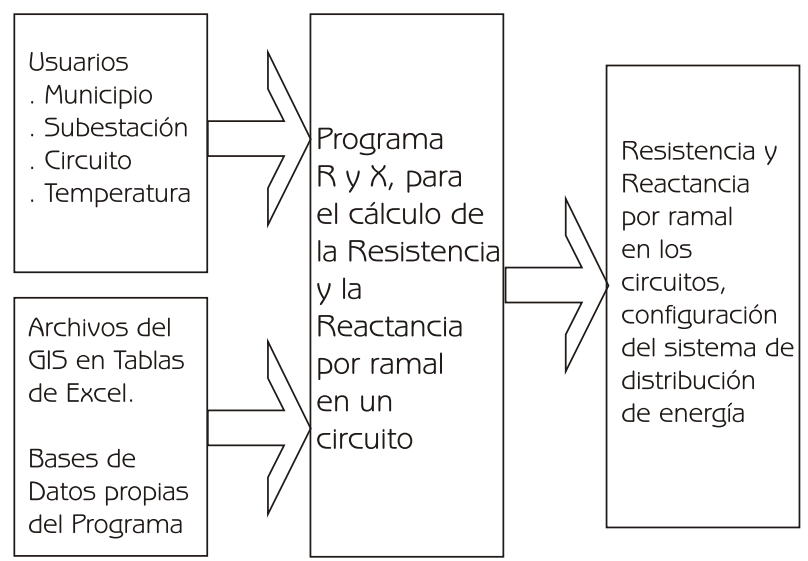

Figura 5. Diagrama de entradas y salidas

Para realizar el cálculo de los valores de resistencia y reactancia se utiliza información de algunas casillas de las tres tablas del SIG y además fue necesario crear en el programa dos bases de datos que son:
1.DEQ_COMJ: Contiene todos los datos sobre los diferentes conjuntos, identificando los espaciamientos reales entre las líneas, el número de fases y el espaciamiento equivalente (Deq).

2. COD_COMD: Contiene la información de la resistencia por metro y el Diámetro medio geométrico para cada uno de los calibres de los conductores de acuerdo al código del SIG.

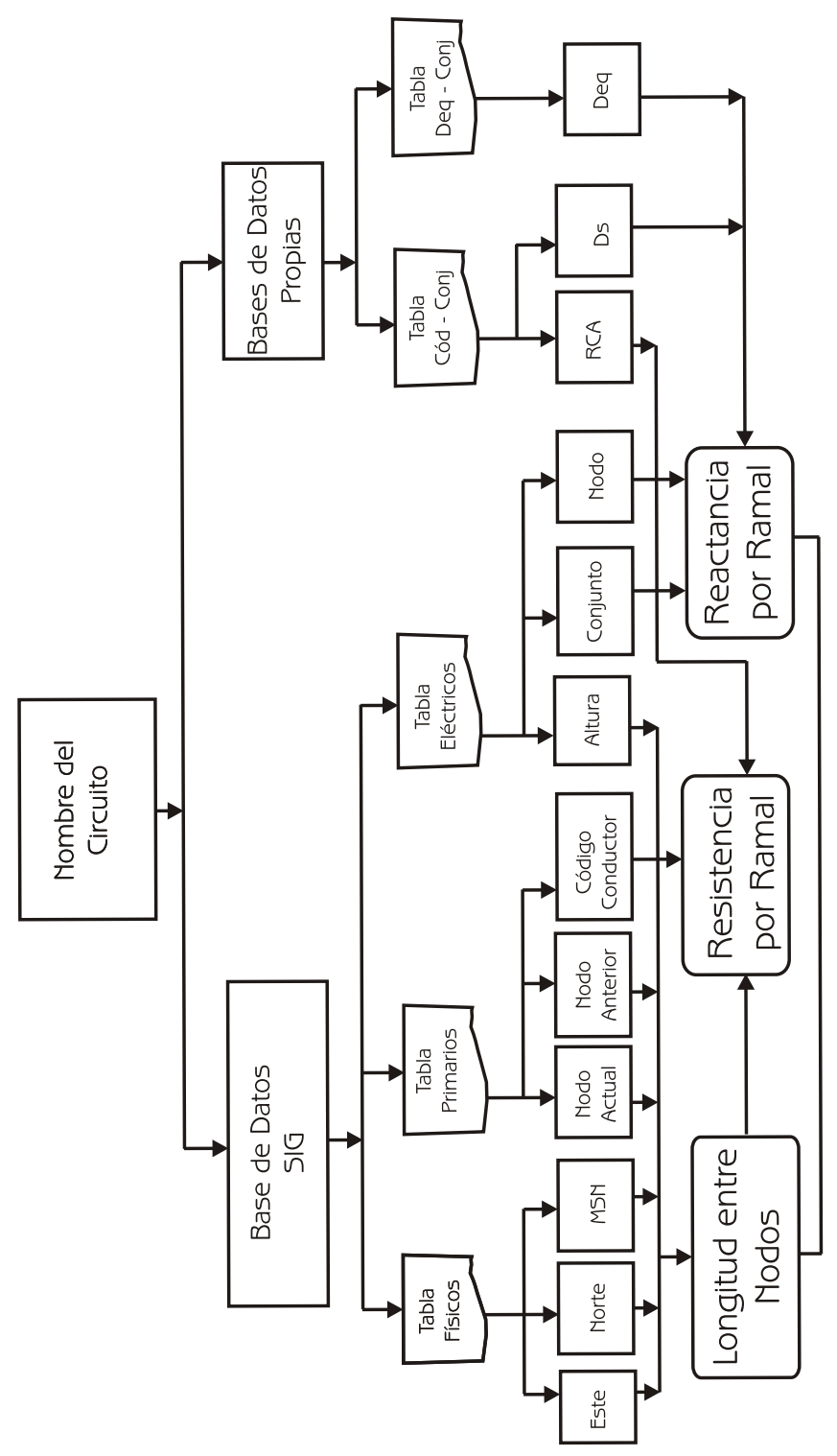

Figura 6. Flujo de Datos del Programa 
En la Figura 6 se ilustran las bases de datos y el flujo de información requerido para el cálculo de los parámetros.

En la Tabla 7 se muestran los resultados del programa para los valores de $R$ y $X$ por ramal por circuito y en la Tabla 8 se ilustra la configuración del circuito.
El programa se probó para los 17 circuitos de la red de distribución de buenaventura, los cuales tienen aproximadamente 300 nodos por circuito. Los cálculos se realizaron con ocho decimales de precisión y fueron comparados con cálculos realizados en forma manual, obteniéndose resultados muy exactos. Además el tiempo de ejecución de los resultados por circuito fue del orden de segundos.

\begin{tabular}{|c|c|c|c|c|c|c|c|c|}
\hline $\begin{array}{c}\text { Nodo } \\
\text { Actual }\end{array}$ & $\begin{array}{c}\text { Nodo } \\
\text { Anterior }\end{array}$ & Código & Faseado & $\begin{array}{c}\text { Longitud } \\
{[\mathrm{Km}]}\end{array}$ & $\begin{array}{c}\mathrm{Rf} \\
(\mathrm{Ohm})\end{array}$ & $\begin{array}{c}\mathrm{Rt} \\
(\mathrm{Ohm})\end{array}$ & $\begin{array}{c}\mathrm{Xf} \\
(\mathrm{Ohm})\end{array}$ & $\begin{array}{c}\mathrm{Xt} \\
(\mathrm{Ohm})\end{array}$ \\
\hline $409381-2$ & $409000-4$ & 08 & RST & 0,0279746 & 0,00998 & 0,02993 & 0,01246 & 0,03737 \\
\hline $409382-2$ & $409381-2$ & 08 & RST & 0,0382988 & 0,01366 & 0,04097 & 0,01620 & 0,04859 \\
\hline $412881-4$ & $409382-2$ & 08 & RST & 0,8411231 & 0,02999 & 0,08998 & 0,03731 & 0,11192 \\
\hline $412641-1$ & $411810-3$ & 11 & RST & 0,4202062 & 0,00806 & 0,02418 & 0,01437 & 0,04310 \\
\hline $412642-1$ & $412641-1$ & 11 & RST & 0,6106798 & 0,01171 & 0,03514 & 0,02088 & 0,06263 \\
\hline $412643-1$ & $412642-1$ & 11 & RST & 0,5168092 & 0,00991 & 0,02974 & 0,01758 & 0,05273 \\
\hline $412644-1$ & $412643-1$ & 11 & RST & 0,4159708 & 0,00798 & 0,02394 & 0,01415 & 0,04244 \\
\hline $412645-1$ & $412644-1$ & 11 & RST & 0,5862703 & 0,01125 & 0,03374 & 0,02004 & 0,06013 \\
\hline $412646-1$ & $412645-1$ & 11 & RST & 0,6254888 & 0,01200 & 0,03600 & 0,02032 & 0,06095 \\
\hline $412647-1$ & $412646-1$ & 11 & RST & 0,6268541 & 0,01203 & 0,03608 & 0,01929 & 0,05788 \\
\hline $412648-1$ & $412647-1$ & 11 & RST & 0,6528623 & 0,01252 & 0,03757 & 0,02009 & 0,06028 \\
\hline $412649-1$ & $412648-1$ & 11 & RST & 0,7163920 & 0,01374 & 0,04123 & 0,02205 & 0,06615 \\
\hline $412650-1$ & $412649-1$ & 11 & RST & 0,6015035 & 0,01154 & 0,03462 & 0,01958 & 0,05874 \\
\hline $409045-1$ & $411810-3$ & 05 & RST & 0,1434652 & 0,00940 & 0,02819 & 0,00674 & 0,02023 \\
\hline
\end{tabular}

Tabla 7. Resultados de los Valores $R$ y $X$ por la Rama por Circuito

\begin{tabular}{|c|c|c|c|c|c|c|}
\hline $\begin{array}{c}\text { Modo } \\
\text { Destino }\end{array}$ & $\begin{array}{c}\text { Modo } \\
\text { Origen }\end{array}$ & Ramal & $\begin{array}{c}\text { Cód. } \\
\text { Ramal }\end{array}$ & $\begin{array}{c}\text { Imax } \\
\text { [Amp] }\end{array}$ & Switche & Estado \\
\hline $409381-2$ & $409000-4$ & 1 & Cto3R1 & 357 & 0 & 0 \\
\hline $409382-2$ & $409381-2$ & 1 & Cto3R2 & 357 & 0 & 0 \\
\hline $412881-4$ & $409382-2$ & 1 & Cto3R3 & 357 & 0 & 0 \\
\hline $412641-1$ & $411810-3$ & 1 & Cto3R21 & 522 & 0 & 0 \\
\hline $412642-1$ & $412641-1$ & 1 & Cto3R22 & 522 & 0 & 0 \\
\hline $412643-1$ & $412642-1$ & 1 & Cto3R23 & 522 & 0 & 0 \\
\hline $412644-1$ & $412643-1$ & 1 & Cto3R24 & 522 & 0 & 0 \\
\hline $412645-1$ & $412644-1$ & 1 & Cto3R25 & 522 & 0 & 0 \\
\hline $412646-1$ & $412645-1$ & 1 & Cto3R26 & 522 & 0 & 0 \\
\hline $412647-1$ & $412646-1$ & 1 & Cto3R27 & 522 & 0 & 0 \\
\hline $412648-1$ & $412647-1$ & 1 & Cto3R28 & 522 & 0 & 0 \\
\hline $412649-1$ & $412648-1$ & 1 & Cto3R29 & 522 & 0 & 0 \\
\hline $412650-1$ & $412649-1$ & 1 & Cto3R30 & 522 & 0 & 0 \\
\hline $409045-1$ & $411810-3$ & 1 & Cto3R31 & 542 & 0 & 0 \\
\hline
\end{tabular}

Tabla 8. Resultados de la configuración del Circuito 


\section{COMCLUSIOMES}

Este artículo presenta una metodología para calcular el modelo de una red primaria a partir de datos actualizados del SIG. Como resultado de la metodología se desarrolló una herramienta computacional que permite calcular en forma automática los valores de resistencia y reactancia de cada una de las ramas, a partir de los datos actualizados de campo. También como parte del modelo se entrega la información de la configuración de la red.

Aunque esta metodología fue aplicada al sistema de distribución del municipio de Buenaventura, puede ser utilizada en cualquier sistema de distribución que tenga información actualizada del SIG y puede ser empleada en sistemas de distribución a nivel de $13.2 \mathrm{KV}$ y $34.5 \mathrm{KV}$ con buena precisión y rapidez de respuesta.

\section{BIBLIOGRAFÍA}

[1] Candelo J, Figueroa D, "Metodología para la recopilación de datos utilizados en la reconfiguración automática de redes de distribución de energía eléctrica". Tesis de grado, Escuela de Ingeniería Eléctrica y Electrónica de la Universidad del Valle, Mayo de 2002, Cali-Colombia.

[2] EPSA S.A E.S.P " Manual de levantamiento de la una red de distribución", Departamento de Sistema de Información geográfica.

[3] Grainger John, Stevenson William, "Análisis de sistemas de potencia", editorial Mc Graw Hill, México 1996. 